\title{
Flow Turbulence And Information Quality
}

Akhilesh Chandra (E-mail: ac10@uakron.edu), University of Akron

Balaji Rajagopalan (E-mail: rajagopa@oakland.edu), Oakland University

Ravindra Krovi (E-mail: krovi@uakron.edu), University of Akron

\begin{abstract}
A crucial aspect of managing the corporate information resource is the ability to assess and maintain information quality. This paper proposes criteria for defining and measuring information quality based on established parameters of information flow. We develop a conceptual model linking information flow metrics and information qualities. The basic premise of the model is that changes in flow metrics affect the usefulness criteria of information, which in turn impact information quality. The usefulness criteria of information are based on established accounting standards. This mapping of flow metrics to information quality is a necessary and critical step towards the development of a robust instrument for quality assessment. The implications of the proposed model for managing information flows resulting from business processes are discussed.
\end{abstract}

"The ability to streamline the structure and to influence and control the flow of information is dramatically more powerful and cost effective than moving and manufacturing physical products. Changing the flow of information requires companies to change not just the product mix, but perhaps more important, the business ecosystem in which they compete. Unless an enterprise develops an explicit strategy to accommodate the accelerated flow of information, the enterprise will find itself scrambling, working harder and faster to stay afloat." (Kalakota and Robinson 1999 p. 6).

\section{Introduction}

1 nformation technology has evolved from a more traditional role of efficiently supporting business processes to becoming one of the primary drivers behind shaping global corporate strategies. Organizations are no longer viewed as an inflexible set of core units; instead they are composed of highly interconnected flexible entities. For some time now, corporations have engaged in various attempts to use technology to streamline their processes. Streamlining involves identifying and eliminating non-value added activities. Companies like IBM that underwent major re-engineering efforts in the nineties have now re-designed their strategies around making business processes E-enabled. Several studies in the business process reengineering (BPR) area have articulated the case for rationalizing organizational processes. In addition to the debate over radical innovation versus continuous improvement (Davenport 1993), some studies have even focused on implementation issues (Davenport and Stoddard 1994).

In the emerging business environment, the focus, however, is not so much on the need to rationalize existing processes but more so to understand the potential implications of these implementations. In this respect, some of the BPR literature has focused on the change aspect that aims at managing organizational resistance to revamp traditional processes (Davenport and Beers 1995; Davenport 1998). However, there is a need to also study the impact of disruptions on the linkages underlying such organizational implementations. An integral part of managing these linkages is to manage the flow of information across vertically oriented systems prevalent in most organizations (Hammer and Stanton 1999).

The need to focus on the flow of information has been espoused by industry leaders such as Bill Gates. In his recent book 'Business @ the speed of thought', Gates argues that contrary to the 1980's and 1990's when the focus was on quality and re-engineering respectively, the 2000's will be about velocity and the flow of information. 
If organizations are to improve their performance using key E-business drivers, then careful attention should be given to understanding how irregularities in information flow affect business processes (Krovi et al. 2002). Such flow irregularities or turbulence can have a serious impact on the quality of information flowing through business processes in an environment marked by integrated functions. The objective of this paper is to develop a conceptual model that depicts the relationship between the properties of information flow and its quality.

Researchers have proposed several measures of information quality. These measures utilize both formal and heuristics based frameworks. Formal frameworks suffer from inflexibility due to their foundation on probability theory (Lea et al. 1992). Similarly, heuristics based frameworks are considered imprecise (Felix and Niles 1988). Some studies have integrated heuristics with quantitative models to evaluate data quality. Using a decision support system approach, Kaplan et al. (1998) proposed an assessment process for auditors to determine the minimum set of controls that should be tested. Their approach combines auditor's heuristics with the model based algorithmic procedures to assess data quality in a client's accounting information system (AIS). In summary, there appears to be only marginal progress on efforts to objectively measure information quality (Walker 1993).

However, by their very nature, data quality assessments in auditing are an after-the-fact event. From a manager's perspective responsible for operational or strategic decisions, there is a need to assess, measure, and influence data quality as information traverses its path along organizational segments. Prior research has made few advances in this regard. The contribution of this paper is to advance theoretical foundations of data quality assessments. The proposed conceptual model provides a basis to examine the effect of changes in flow related metrics on the qualitative features of information.

The rest of the paper is organized along the following lines. Section 2 briefly examines the impact of flow irregularities on business processes, and summarizes the information flow parameters that are considered in this paper. Section 3 discusses the qualitative features of information using the FASB and IASC frameworks. This is followed by the development of the flow-quality model along with illustrations in section 4 . Section 5 presents a discussion of the model and its implications for improving business processes. Finally, concluding remarks are offered in section 6 .

\section{Information Flow}

For the success of partnerships and alliances in business-to-business electronic commerce, it is imperative that information flows smoothly and quickly through the supply chain. Information flows are especially critical given the attempts to electronically link end-customers with retailers, distributors, manufacturers, and suppliers. Smooth integrated information flows across the supply chain are essential for clarity between production information and demand information. Maintaining seamless-ness in this chain of information flow is vital since manufacturers need to know what retailers are ordering and provide that information to suppliers on real time basis. Order changes can then be easily converted into modified schedules, logistical plans, and warehouse operations (Handfield and Nichols 1999). The preamble to this paper succinctly emphasizes the urgency to streamline information flows for corporate viability. Another motivation for us to study information flow stems from its similarity to fluid flow dynamics documented in prior studies (Chandra et al. 1998; Krovi et al. 2002)

Corporate information can be thought of as flowing through and between organizational entities/divisions and changing in form, content, and preciseness (depending on the user's information requirements) as it traverses its path. Thus, characteristics of organizational information transcend the entire organization and could potentially affect information production, storage, and delivery mechanisms of information. The knowledge of these characteristics is important for understanding and influencing the flow of information as it flows through organizational entities over time. Such knowledge is also useful in designing information systems that effectively meet organization's strategic, tactical and operational goals. 


\subsection{Measuring Flow Turbulence}

Information flow turbulence or irregularities such as delays, volatility, biases, etc., can result from an increased number of nodes through which information is processed and altered. Such irregularities have a direct bearing on the quality of information as it traverses its path from producers to consumers. Hence, a key aspect of treating information as a corporate resource is the ability to maintain and enhance its quality. Corporate data based on poor quality control mechanisms could adversely affect decision-making. Unfortunately, too often companies are poorly organized and under-prepared to manage such complex information flows. For effective process value analysis, there is a need for a model that takes into account the metrics that affect the flow of information.

One model discussed in the literature relates to 'information refineries.' The information refinery (IR) concept is based on the notion that electronically distilling raw material leads to better quality information. It describes the number of processing steps needed to produce information. The IR concept has been applied to areas such as document clustering, electronic publishing etc. (Zack 1996; Clippinger and Kosynski 1989). The refinery concept is primarily focused on the production of information in terms of its quality and consequent impact on decision support.

Despite being useful in several contexts such as data errors, and data noise, the IR concept is restrictive, and hence, less useful for the purpose of examining information flow turbulence. Clearly, in this regard, a major weakness of the IR model is its lack of consideration of the dynamics of information transfer between various nodes: for example, between a web storefront catalog and warehouse inventory database. The IR concept is based on a critical assumption: the nodes themselves can seamlessly exchange/transfer information inputs and outputs. From a real world perspective, this is not true - especially in complex supply chains where each entity has information systems that are proprietary in nature due to differences in platforms, information exchange methods, representations etc. For example, transferring customer order information from a node, which has proprietary procurement software like Ariba, seamlessly into an SAP order processing module is complex because of the disparity in information representation and exchange standards. The more the number of nodes along the information transfer path, the higher the likelihood of such variances and, hence, more the impedance. The IR approach also assumes a lack of information uncertainty, which is a simplistic view of the supply chain environments where, often various entities do not have access to information.

\subsection{A Dynamic Model Of Information Flow}

Based on a dynamic view of information flow, Krovi et al. (2002) proposed a set of parameters of information flow to improve and measure business processes. The following discussion briefly summarizes three flow parameters (velocity, viscosity, and volatility) of their model relevant to this study. The relationship between flow metrics and qualitative measures is established in a later section.

Velocity: In fluid dynamics, the velocity of fluid is a function of the time taken to traverse a given distance. From information flow perspective, velocity represents the number of transactions per unit time arriving at a decision node. For example, the number of orders received per day by an organization could reflect the velocity of information. Also, the number of hits received by a news organization's web site (like CNN.com) immediately following the September 11 attacks on the World Trade Center reflects how organizations have to deal with changes in information velocity. 
Volatility: The type of fluid flow is identified with the help of a Reynolds number that is obtained by dividing the inertia force (which is the external force that acts on the fluid), by its viscous force (which is the internal force that acts on fluid molecules). A low value of Reynolds number is indicative of a laminar flow of the fluid. Alternately, a high value of Reynolds number indicates a turbulent flow of the fluid. Thus, the relation between internal and external forces can be used to describe and predict the changing property of fluids.

Information volatility reflects uncertainty with respect to content, format and/or timing of information. Volatility is a function of external forces (stemming from industry, economy, or global sources). For instance, changes in economic policies or interest rate announcements by the Federal Reserve can affect the operating performance of an enterprise.

The nature of effect these forces have on plans and performance of a company causes the resulting information flows to be either laminar or turbulent. Thus, war in the Middle East, nervous signals due to Enron, Arthur Andersen, and WorldComm scandals, and the continuing uncertainty over war on terrorism, provide fodder for turbulent information flows within and between companies. Double-digit daily exchange rate fluctuations could be characterized as turbulent as they can have a destabilizing effect on the balance sheet of a company. Another example of turbulent flow came from the decline in the earnings of Telecom companies in 2000 that prompted fears of spending cuts with consequent effect on equipment manufacturers (such as Cisco Systems, Nortel Networks, etc.). This type of cascading effect is attributed to the symbiotic relationship between the telecom carriers and equipment suppliers - survival of equipment manufacturers depends on spending by Telecom companies.

Viscosity: In fluid dynamics, fluid is composed of molecules that constantly interact with each other. Such interaction is the cause of friction between fluid particles. The property by which a fluid can resist deformation is referred to as viscosity. From information flow perspective, viscosity reflects the inherent conflict faced by a decision maker or information processing node. The conflict is due to the counteracting pieces of information called 'information particles' (IP). An IP is atomic in that it can exist independently and still retain the characteristics of information. In cases of conflict, viscosity appears in the form of multiple values of information that must be resolved before the node can begin processing.

A later section discusses the relationship of these three parameters to the qualitative features of information (discussed below). The resulting model has implications for improving business processes, providing efficient decision support for management and designing effective information systems.

\section{Qualitative Features Of Information}

Qualitative features of accounting information described in this section are based on usefulness considerations as initially defined by the FASB and latter adapted by the IASC. Concept Statement (CON) No. 2 is the primary document of the FASB framework that details specific characteristics of accounting information. Two key features identified by FASB pertinent to decision usefulness are relevance and reliability (Kaplan 1998). Efforts at harmonization of accounting standards by the IASC have also emphasized similar qualitative features of accounting information.

FASB's conceptual framework uses relevance and reliability as broad distinguishing features of accounting information. Relevance ensures that information relates to the decision. Reliability ensures that information is a true representation of the economic transaction. Each of the features is further sub-divided into specific components to assist in the generation and evaluation of information and the design, maintenance and audit of accounting information systems. The conceptual framework also recognizes that organizations do not have the luxury of generating and maintaining all and every information flow. Therefore, relevance and reliability criteria are subject to two business constraints: value analysis and materiality. The former evaluates the value proposition of generating and maintaining the information flow. The latter constraint evaluates the relative significance of information flow vis-à-vis other information flows. Information flow with a positive net value and appropriate significance to influence decision is considered optimal in maintaining efficiency and effectiveness. 
Figure 1 provides definitions of various qualitative features of information. Figure 2, adapted from CON2, briefly sketches a framework of the qualitative features of accounting information.

\section{Figure 1: Properties of information as defined in CON2}

Relevance: The capacity of information to make a difference in a decision by helping users to form predictions about the outcomes of past, present and future events or to confirm or correct prior expectations.

Reliability: The quality of information that assures that information is reasonably free from error and bias and faithfully represents what it purports to represent.

Timeliness: Having information available to a decision-maker before it loses its capacity to influence decisions.

Verifiability: The ability through consensus among measures to ensure that information represents what it purports to represent or that the chosen method of measurement has been used without error or bias.

Neutrality: Absence in reported information of bias intended to attain a predetermined result or to induce a particular mode of behavior.

Predictive Value: The quality of information that helps users to increase the likelihood of correctly forecasting the outcome of past or present events.

Feedback Value: The quality of information that enables users to confirm or correct prior expectations.

Representational Faithfulness: Correspondence of agreement between a measure or description and the phenomenon that it purports to represent.

Figure 2: Qualitative Feature Of Information

(Source: Adapted from FASB, Concept Statement No. 2, 1987)

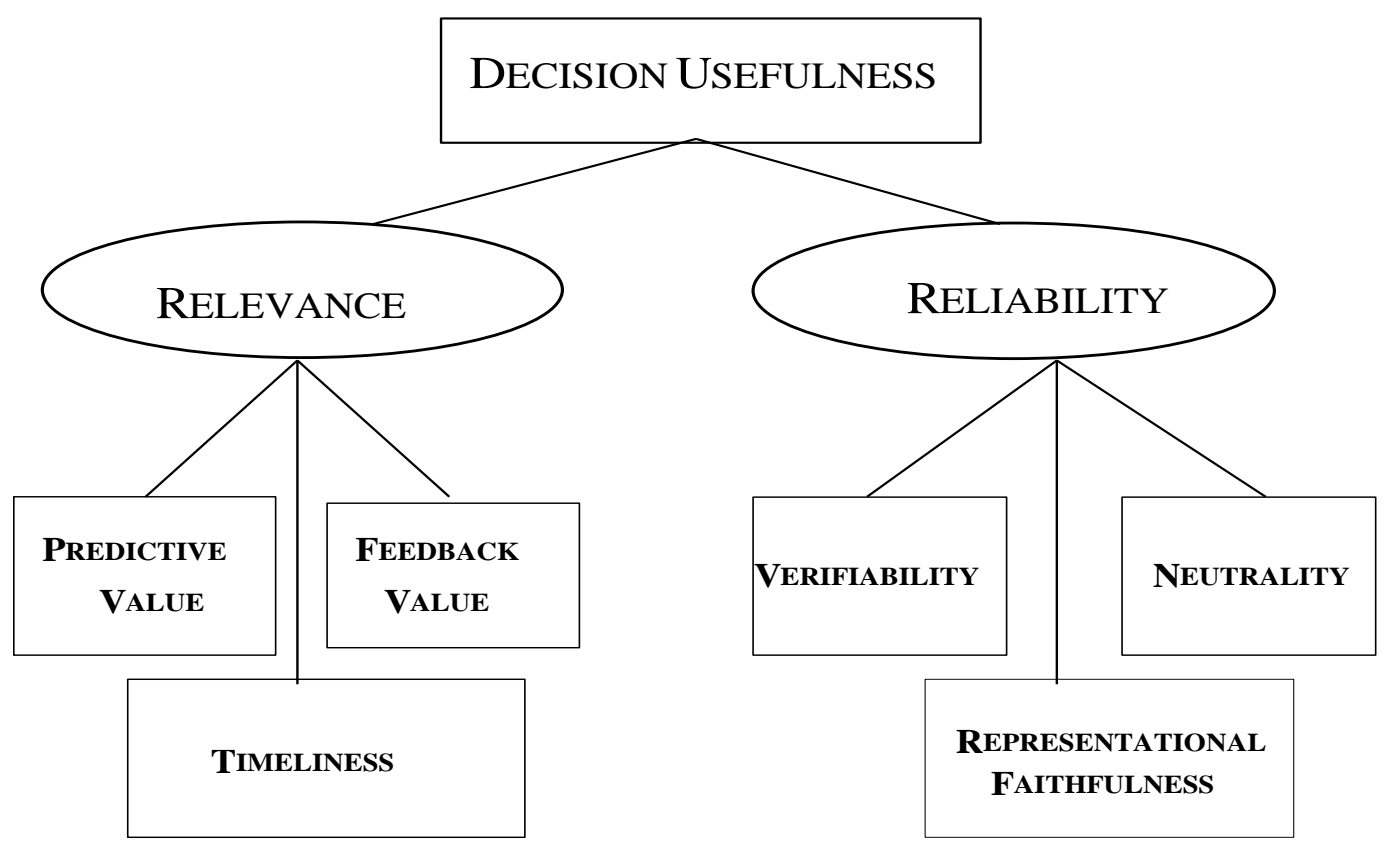


Later IASC adapted these features in developing its own framework aimed at harmonization of accounting standards across countries. Minimizing the diversity of accounting practices amongst member countries resonates in IASC's existing accounting standards. IASC's framework for the preparation of financial statement also emphasizes key qualitative characteristics of accounting information (Muis 1999). These include understandability, relevance, reliability, and comparability. These features are subject to constraints of timeliness, balance between benefit and cost, and balance between qualitative characteristics. As evident, for the most part the qualitative features identified by IASC are similar in import and emphasis to their US counterpart as stated by the FASB. Overall, the qualitative model represents the decision-usefulness paradigm for external reporting.

Albeit described in the context of accounting information for external reporting, the qualitative characteristics set forth by FASB and IASC can be equally applied to all types of corporate information. A closer analysis of the qualitative properties reveals that essentially the same attributes are required for making internal decisions by the management at various levels in an organization. The generic nature of these qualitative features makes them ideal candidates for their universal application in any decision-making situation.

The qualitative criteria serve as a collective indicator of decision usefulness for those who process and use information. The usefulness paradigm stipulates that information must be useful for decision making in general. The relative importance of these qualitative features may vary with the decision context. For instance, the characteristics of information required for strategic decisions are different from those for operational and tactical decisions. Hence, the usefulness criteria can be construed as pointers to the quality of information for various levels of decisions.

However, in evaluating information quality, the qualitative features by themselves provide little help in objectively assessing alternatives. Further, the conceptual nature of the usefulness criteria is subject to interpretation, which may lead to ambiguity in the assessment of information quality. It is important that qualitative features are measured by clearly defined indices to effectively assess and manage information quality. Information systems would be more effective when designed based on measures that affect the flow of information. However, there is no documented effort at relating flow metrics to information quality. The following section identifies such metrics and describes their affect on qualities of information. Specifically, the discussion demonstrates that the usefulness criteria are affected by the metrics of information as it flows through various organizational entities.

\section{Flow Metrics And Qualitative Attributes}

The model proposed in this section predicts changes in qualitative characteristics of information as it traverses its path over time through the organizational network. The flow metrics are defined and derived based on measures used in the analysis of fluid flows. In the absence of flow metrics, the measurement of information quality becomes a function of experience and judgment.

Figure 3 illustrates the relation between the metrics of information flow (as determinants of relevance and reliability) and information quality. The following discussion explains how each of the metrics (i.e., velocity, volatility, and viscosity) relates to information quality via relevance and reliability. 
Figure 3 : Flow-Quality Model

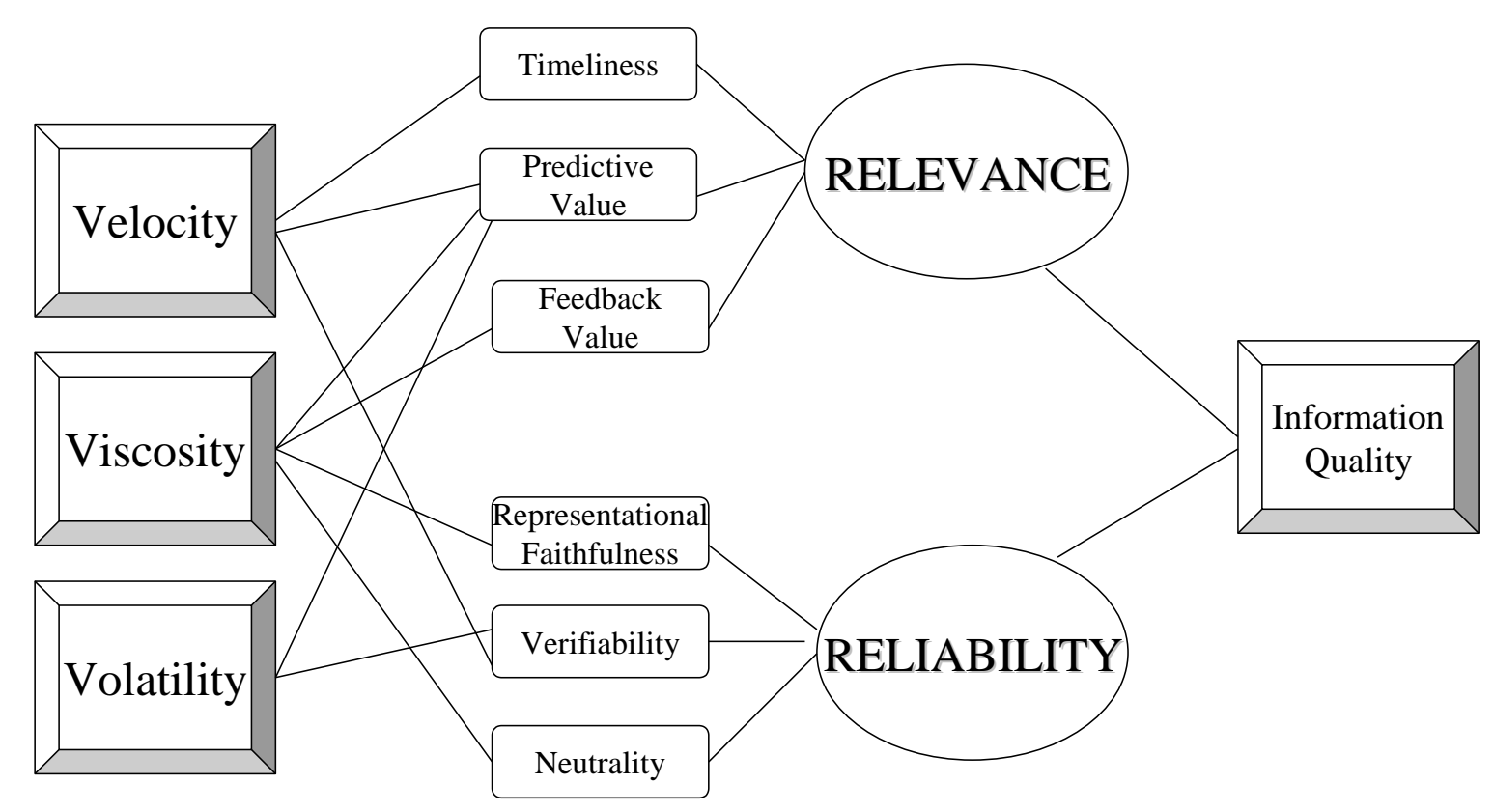

\subsection{Information Velocity}

The speed with which information reaches a decision node affects information quality. The traversal speed varies according to the type of information flow. Three broad types of information flow exist in an organization.

The first form of information flows through formal communication channels. Managers frequently communicate organizational policies, rules and regulations to employees. Such communication takes place either through general memorandums affecting organization wide work force, or specific reports aimed for an individual or group of them in a division. Information transfer from managers to employees containing organizational directives is often uni-directional in nature. This type of information transfer is achieved through formal communication channels along the organizational hierarchy. The wider impact of information carried in formal channels and often the immediacy of its implementation necessitates that such information is communicated to affected parties without loss of time. Thus, the number of nodes through which information traverses may not materially affect the speed of information flow along the formal hierarchy.

The second form of information flows along the informal communication channels. Such information may be less organized (and possibly less precise) than its formal counterpart. The possibility of distortion in the original information is a function of number of nodes in the informal channel. Nodes may also have a negative effect on the speed of information flow. The origin of information in the informal channel is either in response to the information content of the formal communication system, or a function of organizational dynamics. Thus, the speed per unit time of such information would also be influenced by its perceived importance by the affected interested groups.

A third form of information flow occurs between peers. Information between peers can travel along both formal and informal channels. In view of the reduced number of links between the sender and the receiver, information flow among peers is expected to travel at a faster rate per unit time and may be less prone to distortion.

Information velocity within and between the three forms of flows described above is moderated by the frequency and strength of changes affecting the information system, organizational response to those changes, and the belief function held by decision makers. Viewed in the context of a contingency framework, the response 
function will vary between internal and external sources of changes, and the information requirements of those affected by changes. Thus, information requirements of internal managers typically revolve around budget analyses, cost trends, and performance metrics. Similarly, stakeholders outside the organization are more interested in liquidity, solvency and overall profitability of the organization.

Based on the form and source of information flow, velocity affects relevance (via its effect on timeliness and predictive value), and reliability (via its effect on verifiability). Holding other variables constant, timeliness varies directly with velocity. With a fast traversal speed, information would reach the decision maker in a timely manner to help him/her make the decision. The following example further exemplifies the relationship.

\section{Production Plan Example:}

For illustration purposes, consider the case of a production manager whose task is to schedule the number of units to produce. Production plans are a function of marketing forecasts. Such forecasts can be either internally generated or obtained from independent outside agencies. Further, depending on the size of organization, such information may have to be compiled and aggregated from its sales force. The hierarchy of organizational set up in its sales force would determine the nodes through which information would traverse to reach the marketing manager.

If marketing department delays in obtaining, compiling and sending sales forecasts figures to the production manager, it will adversely affect production plans pertaining to hiring of labor, purchasing of raw materials, and scheduling of plant and machinery. The lack of timeliness will translate into delayed or missed customer order. The slow speed of information traversal would also affect the precision with which change in raw material can be predicted. Change in economic conditions (external stimulus) when combined with slow speed of internal information traversal may create uncertainty to make accurate predictions. If information reaches a node at a slower rate, then it loses its predictive power for a decision context with a strategic focus. Thus, by affecting its timeless and predictive value, information velocity determines the relevance of information.

Velocity and reliability are inversely related in this example. Slow arriving information affords greater possibility to verify its accuracy, thereby, increasing reliability of information. In the alternate case of high velocity of information, organization would have to invest in stringent online internal controls to make up for the same level of assurance available in case of slow traveling information. Information that arrives at a faster rate may be less valid because of the time constraint in verifying its content. This inability might translate into the sender's bias, thereby, corrupting the information and reducing its reliability.

\subsection{Information Volatility}

The volatility of information flow is identified as either laminar or turbulent. The two types are distinguished by the relative variance between ex-ante and ex-post information uncertainty. Small variance defines a laminar flow; a large variance defines a turbulent flow. Variance is influenced by organizational dynamics, decision context, decision time frame, risk tolerance, and belief function of decision makers.

The ratio of external and internal forces acting on the organization measures the type of information flow. The external force pertains to either industry-wide or economy-wide factors. Empirical evidence suggests that macro-economic variables affect the expected cash flows of the organization. The nature of external forces makes it difficult for the organization to control the timing, content and, likely impact of the related information. Therefore, the adaptive response mechanism is critical for organizations and its agents to reach and adjust to environmental information uncertainty. A proactive strategic approach (subject to acceptable risk tolerance levels) should reduce the flow turbulence within conservative margin of error values. Thus, a laminar flow of information between marketing and operating activities could translate into higher quality information via timely and precise sales forecasts. 
Managers incorporate the signals generated from external forces to affect (and/or revise) current and future plans. Similarly, investors or creditors revise their expectations about future cash flows of an organization based on economy and industry wide factors. External forces may generate varying information flows. A laminar (turbulent) flow of information should be accompanied with even (uneven) variance. A turbulent information flow would have less predictive power; while a laminar flow of information would have definite patterns to form, conform, or change expectations about cash flows. Thus, the flow type directly affects the relevance of information. For instance, turbulent nature of information affects the content of information and the speed with which it reaches the destination. Turbulence also increases the error in information measurement. This could reduce the ability to verify a transaction. As a result, a turbulent flow could adversely affect the reliability of information.

\section{Production Plan Example:}

Consider the case of the production manager again. The internal force reflects the degree of variability between production and sales. External force is represented by industry and economy wide variables. Prevailing general economic downturn, continuing war on terrorism, mismanagement in financial and capital markets, and collapse of Enron and WorldCom (and associated events) translate into difficulty of minimizing variance between actual and expected levels of raw material. This difficulty would be reflected in weak predictive power of production plans, and reduced information relevance for making precise decisions.

Larger and frequent variability in major economic indices (due to current fluctuating stock market) also affects a steady flow of funds. The consequent increase in the error in information measurement adversely affects information verifiability and hence the reliability.

\subsection{Information Viscosity}

The concept of information particle was introduced in an earlier section to signify the smallest component of information that resists changes to its character when considered individually. The IPs may be affected by changes in data, information flow, environment, and by other IPs.

As an illustration, consider the case of inventory appearing on the balance sheet of a company. Management has to balance potentially conflicting demands on holding inventory: an inventor balance of less than the optimum may result in lost sales and poor customer goodwill. Alternately, an inventory balance of more than the optimum increases costs via storage, insurance, theft, and management expenses.

This example has two IPs:

1. Holding less inventory may result in lost sales, and

2. Holding excess inventory represents an under-utilization of assets.

Each of the two IPs can be used independently in any decision context. Information viscosity is affected by the interaction of such IPs. IPs may either contradict or support each other.

Any resulting friction between two IPs is the source of resistance to an attempt to adapt them to a decision making task. In the previous example, the two IPs (viz., 'holding less than optimum inventory' versus 'holding excess inventory') are conflicting pieces of information. This property of inherent conflict between IPs makes it less flexible for their user to make effective decisions. Reduced flexibility increases the viscosity of information flow and makes it less possible to change the character of information. The contradictory nature of IPs in the previous example makes it difficult to decide about the optimum level of inventory holdings. Alternately, IPs supporting each other reduce the viscosity of information flow by enhancing the flexibility to make decision. Thus, the property of viscosity directly affects the 'relevance' of information by affecting the feedback value and predictive value. 
The effect of viscosity on information reliability appears to be indirect. An IP being the constituent of information has the ability to appropriately describe that information. This ability may be affected adversely if IPs are contradictory to each other representing a situation of high viscosity. Another effect of the contradictory nature of IPs is the relatively reduced ability to bias information in any one direction. These properties accrue due to less conflict between IPs resulting in more resistance to changes. Thus, viscosity affects reliability of information by influencing neutrality and representational faithfulness.

\section{Production Plan Example:}

Consider the case of production manager for one last time. Two classes of IPs are pertinent in this case: optimum balance of raw material inventory and optimum number of labor force. Together the two IPs determine the optimum balance of work in process and finished goods inventory. Assuming relatively predictable economic conditions (representing external forces), the conflict within and between each of the two classes of IPs is likely to be minimal. The reduced conflict should increase the predictive power and feedback value with respect to the level of raw material inventory and strength of work force. The content and character of information will be less vitiated in the event of IPs supporting each other. This will enhance representational faithfulness and increase neutrality about production plans (by reducing the possibility of bias in any one direction by the production manager). Thus, the greater cohesiveness between IPs seems to have a positive effect on both relevance and reliability.

\subsection{The Importance Of Flow Metrics: An Illustration}

Business process efficiencies can be realized through effective management of complex information flows. Traditionally, information is studied mostly in terms of its decision usefulness qualities (viz., relevance and reliability).

Information quality is influenced by the quality of organizational data and business processes. Given that planning is a fundamental activity in any organization and planning assumes information uncertainty (due to its forward looking nature), the length of planning time frame is expected to be inversely related to quality of data and business processes. This conclusion is readily apparent when we consider the chain reaction information uncertainty (in planning process) feeds into qualitative variables. A longer term forward planning has heightened uncertainty levels with relatively reduced predictive value (and information relevance) and makes the information flow relatively turbulent in nature. In the face of uncertainty, it is challenging to accurately and reliably verify facts with a high degree of assurance about information accuracy and its decision usefulness. The end result of these cumulative successive and interdependent events in this chain (triggered by environmental uncertainty) is to impair information quality (via reduced relevance and reliability).

The following discussion illustrates how the study of flow metrics can foster an understanding of qualitative features of information and its implications for assessing information quality. A simplified AIS model is used to illustrate related business processes with the help of Figure 4.

The figure consists of information flows between basic transaction cycles. Market forecasts trigger information generation that is fed through planning, expenditure and production transactions. The information is provided to both external and internal users. The users of information provide feedback that helps in modifying the plans. While the discussion that follows is context sensitive, its implications are valid across various organizational functions. 
Figure 4: Information Flows In A Simplified AIS

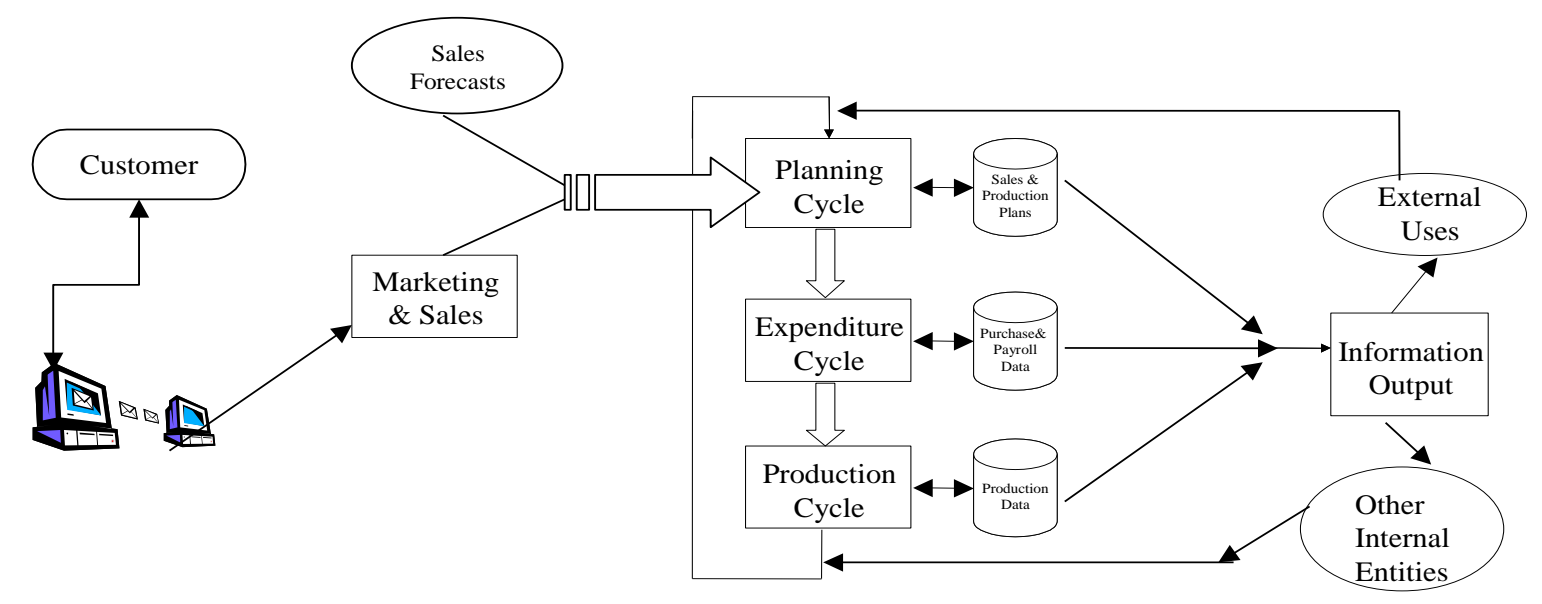

A typical online transaction begins with customers placing orders via a company's website. A relative surge in customer orders defines velocity in this context. The nature and complexity of the traversal path from customers to the organization affect the relative velocity of information flow. The impact and urgency in an online environment on the back-end logistics is both direct and immediate. A poorly designed and outdated information system (such as that featured by legacy systems and non-connected modules of various ERP systems, for example) would effectively translate into a mismatch between customer orders arriving at company's portal with a high velocity, and piled up and back-logged logistics at the back end (crawling at a very low velocity).

Unless organizations take proactive and remedial actions (via properly streamlining and linking desperate systems), there is a certain danger that the mismatched flow velocity could adversely affect operational performance and customer goodwill (as was evident in the case of ToysRUs and Hersheys in 1999). Other proactive measures include performing stress tests of site and server reliability, taking into account the number of orders taken, transactions processed, or information requests generated. Besides internally generated changes, increase in velocity can be manifested by external events as well. For example, unsettling events in the Middle East could trigger a precipitous drop in the market. This may cause an increase in incoming traffic at electronic trading sites.

Online orders also have an impact on fulfillment systems particularly during the holiday season. This requires an understanding of the impact of volatility of generated demand vis-à-vis incoming traffic on background operations. The flow quality will be affected by how well the front-end operations are seamlessly integrated with back-end operations. Integration eventually affects the ability of the site to make 'availability to promise' versus 'availability to deliver' guarantees.

Integration also affects the credit check function. On receipt of the sales order, the company would follow them up by processing the orders through credit and inventory check. Depending on the strategic objectives, a company may choose to outsource the credit check function. However, when business process outsourcing alternative is adopted, companies must invest in systems integration (between company's business processes with those of partner's business processes) in order to appropriately optimize flow velocity. Lack of proper integration and a large number of orders per unit time would increase uncertainty, and reduce relevance and reliability of information. Higher uncertainty may result in delay in performing relevant checks, and adversely affect the feedback and forecasting value and the timeliness of the process expected by the customer. 
Another factor affecting information quality is the diversity of a sales order and its match with the organization of inventory in the warehouse. A sales order could include any combination of several kinds of inventory (distinguished by slow, medium or fast moving items). The resulting variability in processing cycles for each inventory product in the order triggers an erratic and unpredictable flow of information - which is of turbulent nature. Therefore, a delay in processing orders due to incompatibilities of sales orders with physical inventory organization reduces information viscosity. Reduced viscosity dampens the relevance of information flow especially when sales orders are time sensitive (as is the case during holiday sales).

\section{Implications \& Conclusions}

The implication of flow-quality framework for information can be analyzed from the standpoint of management control and systems design. From the perspective of management, knowledge of factors that affect information quality assumes significance in efficiently and effectively providing decision support. The proposed model provides directions for developing objective guidelines for assessing the qualitative features of information established by regulatory bodies. For example, 'relevance' is a significant criterion of corporate information. However, it would be useful if the user has an objective process to measure relevance. Such measurement would minimize subjective evaluations of relevance across decision contexts. The development of an index or a quantitative metrics to uniformly interpret and measure relevance would facilitate a neutral but objective evaluation process that is independent of the variances of decision situations.

System designers also need to take these metrics into consideration to increase the decision usefulness of information for users. Information systems should incorporate the effect of flow parameters in suitably modifying information quality pursuant to information needs of the decision maker.

The proposed model is an initial but necessary and critical step towards attaining precision in quality factors. Future extensions include development of empirical studies (in different domains) to test the precision and effectiveness of relationship identified in this paper.

\subsection{Conclusions}

Corporate information systems play a crucial role in organizational processes. Management of the corporate information resource is vital to organizational success. As such, adequate controls should be in place to ensure that the information processed by these systems is of high quality. However, quality is a relative term due to varying perceptions of what exactly constitutes an important construct. This problem is particularly evident given FASB's conceptual framework of different qualitative features. A better understanding of the corporate information life cycle is a prerequisite for organizations to successfully implement quality programs and tools. This study takes an innovative view of the problem of quality assessment in corporate information systems. Specifically, the focus in this paper is at the information and knowledge level to assess data quality. The advantage of this approach is that it relies on widely accepted guidelines set by FASB and IASC.

In this regard, we have developed a conceptual model that illustrates how information flow metrics affect quality. Although illustrated in the accounting context, the flow-quality model advocated in this paper is generic enough to be applicable to any type of information. This is a critical step towards understanding how metrics of information flow affect qualitative features of information that in turn impact information quality. Future research will focus on refining and extending the model, testing and validation. 


\section{References}

1. Anonymous, (1999) "Information flow, accuracy, timeliness keys to future success", Modern Materials Handling, 54, 7, 7-9.

2. Ballou, D Wang, R Pazer, H and Tayi, G K (1998) "Modeling Information Manufacturing Systems to Determine Information Product Quality", Management Science 44 (4) 462-484.

3. Batchelor, G K (1967) An Introduction to Fluid Dynamics. Cambridge University Press, London.

4. Chandra, A., R. Krovi, and B. Rajagopalan. (1998). "Flow Parameters and Quality in Accounting Information Systems", Massachusetts Institute of Technology (MIT) Conference on Information Quality, October 23-25: Boston, MA.

5. Chen, C R Lin, J W and Sauer, D A. (1997). "Earnings Announcements, Quality and Quantity of Information, and Stock Price Changes", Journal of Financial Research 20 (4) 483-491.

6. CON 2 (1980) "Statement of Financial Accounting Concepts No. 2 - Qualitative Characteristics of Accounting Information", Financial Accounting Standards Board McGrawHill: New York.

7. Felix, W. L. Jr. and M. S. Niles. "Research in Internal Control Evaluation", Auditing: A Journal of Practice and Theory. (Spring 1988), 43-60.

8. Hammer, M., and Stanton, S. "How process enterprises really work", Harvard Business Review, 77, 6 (Nov/Dec 1999), 108-118.

9. Handfield, Robert and Nichols, Ernest (1999) Introduction to Supply Chain Management. Prentice Hall, Upper Saddle River, New Jersey.

10. Hau, L. L., Padmanabhan, V., and Whang, S. "The Bullwhip effect in Supply Chains", Sloan Management Review, Spring 1997, 93-102.

11. Huarng, A S and Krovi, R (1998) "An Object-Based Infrastructure for Information Resource Management", Journal of Information Systems Management. 46-52.

12. IASC (1999) A Comprehensive Standard on Financial Instruments. UK: 5.

13. Kalakota, Ravi and Robinson, Marcia (1999) E-Business: Roadmap for Success. Addison Wesley, Reading MA.

14. Kaplan, D Krishnan, R Padman, R and Peters, J (1998) "Assessing Data Quality in Accounting Information Systems", Communications of the Association of Computing Machinery. (41) 2 72-78.

15. Krovi, R., A. Chandra, and B. Rajagopalan. 2002. "Information Flow Parameters for Manageing Organizational Processes", Communications of the Association of Computing Machinery. Forthcoming.

16. Lea, R. B., S. J. Adams, and R. F. Boykin. 1992. "Modeling of the Audit Risk Assessment Process at the Assertion Level Within an Account Balance", Auditing: A Journal of Practice and Theory, (Supplement). 152-179.

17. Muis, J W Vice President and Controller, The World Bank (1999) on Global Accounting, Auditing, and Financial Architecture, at an SEC conference on 12 April 1999.

18. Orr, K (1998) "Data Quality and Systems Theory", Communications of the Association of Computing Machinery. (41) 2 66-71.

19. Redman, T C (1998) "The Impact of Poor Data Quality on the Typical Enterprise", Communications of the Association of Computing Machinery. (41) 2 79-82.

20. Walker, W. S. "Auditors' Assessments of Inherent and Control Risk in Field Settings", The Accounting Review, 68, 4 (1993), 783-802.

21. Wang, R Y (1998) “A Product Perspective on Total Data Quality Management”, CACM (41) 2 58-65.

22. Wang, R Y and Strong, D M (1996) "Beyond Accuracy: What Data Quality Means to Data Consumers", Journal of Management Information Systems. (12) 4 5-34.

23. Zmud, R W Lind, M L AND Young, F W (1990) “An Attribute Space for Organizational Communication Channels", Information Systems Research. (1) 4 440-457. 
Notes 